\title{
INVESTIGACIONES
}

\section{Divergencias socioculturales en el uso del castellano: repercusiones en la comprensión del alumnado inmigrante en el sistema educativo español*}

\author{
Sociocultural differences in the use of Spanish: \\ repercussions in immigrant student's comprehension in the Spanish educational system
}

\author{
Elena Martín-Pastor, ${ }^{a}$ Francisca González-Gil, ${ }^{a b}$ \\ Cristina Jenaro Río, ${ }^{c}$ Susan Sanhueza, ${ }^{d}$ Patrick M. Pascal ${ }^{d}$ \\ ${ }^{a}$ Facultad de Educación, Universidad de Salamanca \\ Telf.: (34) 923294630. Correo electrónico: emapa@usal.es \\ bTelf.: (34)923294630. Correo electrónico: frang@usal.es \\ 'Facultad de Psicología, Universidad de Salamanca \\ Telf.: (34) 923294610. Correo electrónico: crisje@usal.es \\ ${ }^{\mathrm{d}}$ Universidad Católica del Maule
}

\begin{abstract}
RESUMEN
Este trabajo tiene por objetivo analizar semejanzas y diferencias en la comprensión de textos escolares, y su consecuente repercusión en el aprendizaje, motivadas por divergencias en el uso social y cultural del castellano de estudiantes de origen inmigrante, incorporados al sistema educativo español, frente a sus compañeros españoles de Educación Secundaria Obligatoria (ESO). Se contó con la participación de 402 alumnos de Salamanca (España), de los cuales 196 procedían de otros países, a los que se les administró un cuestionario ad hoc de preguntas abiertas, referidas a textos extraídos de los libros con los que trabajaban en sus clases. Los resultados revelan la existencia de diferencias significativas en dicha competencia con peores puntuaciones por parte del alumnado extranjero justificadas, en parte, por la influencia que ejerce la cultura sobre la que se sustenta lo hablado y escrito, y no tanto por un desconocimiento de la lengua vehicular de la escuela.
\end{abstract}

Palabras clave: escolares extranjeros, diversidad lingüística y cultural, inclusión, comprensión lectora, ESO.

\section{ABSTRACT}

Our study aims to analyze the similarities and differences in reading comprehension, due to the influence of sociolinguistic and sociocultural components among immigrant students that study in the Spanish educational system, as compared to their peers in compulsory secondary education. To test this, a sample of 402 students from Salamanca (Spain), of which 196 students were immigrants, was selected. An ad hoc reading comprehension measure developed for the current study was used for the assessment. The results obtained show significant differences in understanding; immigrant students have more difficulties that can be attributed, in part, to the culture of the spoken and written language and not because they don`t speak the language.

Key words: immigrant students, cultural and linguistic diversity, inclusion, reading comprehension. 
Estudios Pedagógicos XLIII, N 2: 179-193, 2017

DIVERGENCIAS SOCIOCULTURALES EN EL USO DEL CASTELLANO: REPERCUSIONES EN LA COMPRENSIÓN

DEL ALUMNADO INMIGRANTE EN EL SISTEMA EDUCATIVO ESPAÑOL

\section{INTRODUCCIÓN}

La presencia de alumnos extranjeros en el sistema educativo español ha convertido las aulas en contextos pluriculturales y heterogéneos. Sin embargo, a pesar de que se ha convertido en una realidad escolar cotidiana, aún nos encontramos ante la necesidad de diseñar una respuesta pedagógica que se ajuste a esta situación; una educación inclusiva que fomente el crecimiento académico partiendo de la diversidad que nos encontramos en el aula (Pérez \& Sarrate, 2013; Sánchez, Navas \& Holgado, 2013; Santana, Feliciano \& Jiménez, 2015; Simó et al., 2014). En este sentido, Brind, Harper y Moore (2008) afirman que las escuelas son eficaces cuando son sensibles a las identidades de todos los estudiantes y hacen esfuerzos por incluir en el currículum sus historias, intereses, idiomas, religiones y culturas. De hecho, Pérez y Sarrate (2013) señalan que un proyecto educativo deber ser, por definición, intercultural.

En este escenario, nuestro trabajo se centra en el alumnado inmigrante al ser uno de los colectivos a los que con mayor frecuencia se le atribuye un bajo rendimiento académico, dificultades de aprendizaje y altas tasas de fracaso y abandono escolar (Gregorio \& Prado, 2016). Distintos autores (Carvallo, Caso \& Contreras, 2007; Darmody, Byrne \& McGinnity, 2014; García, Rubio \& Bouachra, 2008, 2015; Huguet \& Navarro, 2003; Huguet, Navarro \& Janes, 2007; Prado, 2011; Sansó, Navarro \& Huguet, 2015; Stanat \& Christensen, 2006) aluden a una serie de factores que contribuyen a explicar esta situación: la edad de llegada e inicio de la escolarización, la lengua materna versus la lengua vehicular de la escuela, la trayectoria educativa, las características del entorno social y escolar de acogida, la desigual distribución de estos alumnos en centros públicos y privados o las actitudes y la formación del profesorado.

Por otro lado, vivimos en una cultura letrada donde la palabra, en sus múltiples formatos (oral, escrita, digitalizada) es el principal vehículo de interacción. En consecuencia, la lengua es la manifestación que cada comunidad adopta para comunicarse a través de un código propio y que difiere de unos contextos (geográficos, sociales, culturales, lingüísticos) a otros, al tiempo que es un instrumento para el desarrollo y acumulación de experiencias (González, 1987). Por tanto, la diversidad linguiística es una realidad inherente a la lengua y a sus usos sociales por parte de los hablantes. De ello se deriva el reconocimiento de la misma como una entidad variable y condicionada por factores socioculturales y situacionales (Moreno, 2007), y como la única institución que todas las personas deben dominar para funcionar en cualquiera de las áreas institucionalizadas de la vida social, incluida la educativa. Estudios en contextos anglosajones (p.e. Leith, 2012) avalan este hecho, en consonancia con trabajos previos sobre el lenguaje como recurso social, en los que se incide en su relevancia a la hora de promover acciones para las minorías linguiísticas (Bordieu \& Thompson, 2001; Kymlicka \& Patten, 2003).

Desde esta perspectiva, el presente trabajo evalúa, a través de la comprensión lectora, la competencia sociolingüística y las habilidades en el manejo del castellano de los alumnos inmigrantes, entendidas estas como el conjunto de conocimientos necesarios para, más allá de aspectos gramaticales, utilizar la lengua de acuerdo a las exigencias contextuales a nivel social y cultural de un determinado país o zona geográfica (Moreno, 2007), esencial además para que este alumnado pueda de verdad acceder al sistema educativo y transitar por él obteniendo el máximo rendimiento (Besalú, 2011; Brind, Harper \& Moore, 2008; Nusche, 2009; Sansó et al., 2015). No podemos olvidar que la lectura constituye un elemento crítico que determina el éxito escolar de los alumnos en todas las materias (Alanís, 2004). 
En este ámbito, los estudios que han abordado la comprensión lectora se han centrado en analizar la influencia de las características de los alumnos (procesos psicológicos, habilidades lingüísticas, actitudes, conocimientos previos, estrategias metacognitivas...) y las relativas al texto (nivel de complejidad, estructura organizativa...) en esta competencia, tanto en diferentes niveles educativos como con escolares con diferentes características como discapacidad intelectual, sensorial o TDAH (Alvarado et al., 2011; Sacristán, 2006; Salvador, Gallego \& Mieres, 2007; Santos \& Campo, 2008). Sin embargo, en el contexto español no encontramos investigaciones que hayan abordado la repercusión de los elementos culturales del contexto en el proceso lector, desde la perspectiva de la lectura como un proceso complejo, interactivo e interpretativo, cuyo desarrollo está determinado por el contexto social y cultural (Sulzby \& Teale Cit. en Alanís, 2004). Así, partimos del supuesto de que los mediadores comunicacionales de la cultura de un país producen una desigualdad en aquellos estudiantes procedentes de diferentes entornos sociales, lo que genera en ellos una barrera cultural que dificulta su comprensión y, por tanto, su comunicación, sus relaciones y su participación en la sociedad como sujetos activos. En este sentido, Salvador y Gutiérrez (2005) coinciden en que no debemos pasar por alto la relación entre el dominio de una lengua y los factores socioculturales en los que se desarrolla, ya que es un índice del nivel cultural y social de la persona. Por ello, es crucial incluir las experiencias lingüísticas del alumnado inmigrante en todos los aspectos del currículum (Celedón-Pattichis, 2004). Además, teniendo en cuenta que cada grupo humano desarrolla sus propias prácticas discursivas (Cassany, 2006), el conocimiento de palabras y expresiones va a ser crucial para la comprensión lectora (Alanís, 2004).

A este reto se enfrentan diariamente los estudiantes extranjeros procedentes de países de habla hispana quienes, a pesar de compartir la misma lengua que sus compañeros de nacionalidad española, manejan códigos comunicativos diferentes. Esto se refleja de manera especial en la lectura que, como expresa Cassany (2006), se convierte en una "práctica cultural insertada en una comunidad particular, que posee una historia, una tradición, unos hábitos y unas prácticas comunicativas especiales" (p. 38). Nos situamos, por tanto, ante una concepción sociocultural donde el discurso es un reflejo de los puntos de vista del que lo construye frente al significado que atribuimos al mismo a partir de nuestros conocimientos y experiencias acumuladas. Ello nos proporciona, en palabras de Olson (1998), un modelo para el conocimiento. En esta misma línea, Ruiz (2000) afirma que es a partir de ese conocimiento sobre el mundo y sobre nosotros mismos con el que expresamos conceptos e ideas, y que su uso se debe a procesos psicolingüísticos que se dividen en modelos culturales que varían de unos grupos sociales a otros.

Si analizamos estos aspectos en el ámbito escolar, vemos que se hacen más latentes en las materias de Matemáticas y Lengua, donde los procesos de enseñanza-aprendizaje han estado limitados sobre todo por el currículo prescrito y los libros de texto como una "manifestación del conocimiento general oficial, del sentido común y de las ideologías dominantes de la sociedad" (Van Dijk, 2003, p. 210), que se ajustan a los requisitos predefinidos a nivel nacional. Aspecto que también advierten Gregorio y Prado (2016) al afirmar que la escuela selecciona una serie de conocimientos y prácticas y desecha otras. Una muestra de ello es que, por ejemplo, solo las lenguas europeas forman parte del currículum, quedando las orientales, en el mejor de los casos, relegadas a actividades extraescolares (Carrasco \& Gibson, 2010). Estos hechos generan problemas de comprensión en alumnos de otros países y contextos socioculturales (Olson, 1998), ya que, como señalan Salvador 
Estudios Pedagógicos XLIII, Nº 2: 179-193, 2017

DIVERGENCIAS SOCIOCULTURALES EN EL USO DEL CASTELLANO: REPERCUSIONES EN LA COMPRENSIÓN DEL ALUMNADO INMIGRANTE EN EL SISTEMA EDUCATIVO ESPAÑOL

y Gutiérrez (2005), "el hecho de pertenecer a una clase social o a una cultura condiciona, positiva o negativamente, el dominio de la expresión escrita y de la comprensión” (p. 22). Así, nuevamente se pone de manifiesto la necesidad de incluir en el currículum escolar elementos lingüísticos, sociales y culturales propios de las experiencias de todos los alumnos inmigrantes que forman parte del sistema educativo (Alanís, 2004; CeledónPattichis, 2004). Además, si tenemos en cuenta que la lectura y la escritura son un medio privilegiado para otros aprendizajes, dificultades en las mismas pueden acarrear a su vez dificultades en el aprendizaje del resto de las materias escolares.

A nivel internacional, la creación en el año 2001 del Marco común para las lenguas: aprendizaje, enseñanza y evaluación (MCER), constituyó la base para la defensa del plurilingüismo y dar así respuesta a la variedad lingüística resultante del incremento de la inmigración en Europa (Beacco et al., 2010). No obstante, proyectos para su enseñanza en el contexto escolar como "Lenguas de escolarización" o el Plan Curricular del Instituto Cervantes en España, acentúan la enseñanza de aspectos gramaticales de la lengua, a expensas del desarrollo de habilidades comunicativas y el uso social de la misma (García et al., 2008). Ello impide acceder a la cultura que sustenta tal idioma, es decir, a los valores, hábitos, actitudes que caracterizan su uso social. Por tanto, lengua y cultura son conceptos indisolubles y así, el conocimiento de otra lengua supone enfrentarse a un aprendizaje intercultural, en la medida en que aprendamos a respetar y valorar otras perspectivas diferentes a la propia (Prado, 2011).

La mayoría de las investigaciones llevadas a cabo sobre las dificultades en el ámbito lingüístico y comunicativo del alumnado extranjero, se han centrado mayoritariamente en el estudio del proceso de adquisición y conocimiento de la lengua vehicular de la escuela (Huguet, 2008; Huguet et al., 2011; Huguet \& Navarro, 2003; Navarro \& Huguet, 2006; Navarro, Huguet \& Sansó, 2016; Oller \& Vila, 2011). Sin embargo, estos estudios no contemplan el componente sociolingüístico y sociocultural que tiñe al idioma de un país de sus propias peculiaridades discursivas (Prado, 2011) y que puede generar a estos escolares problemas para comprender la intención del emisor al manejar unas expresiones, un vocabulario y una estructura comunicativa que difieren de los propios, lo que desemboca en una limitación o en una barrera para el aprendizaje (González, 1987).

A la vista de lo previamente expuesto, con el presente trabajo pretendemos contribuir a ampliar el estado del conocimiento existente respecto a las dificultades escolares del alumnado inmigrante, más concretamente en cuanto a la influencia de los modelos comunicativos propios de un país (expresiones, connotaciones de significado y formas de utilizar el lenguaje) en su proceso de aprendizaje. Nos planteamos por tanto como objetivo, identificar semejanzas y diferencias en la comprensión lectora, motivadas por divergencias en el uso social y cultural del castellano, de estudiantes extranjeros frente a sus iguales de ESO. Hipotetizamos además que (1) existirán diferencias en esta competencia (Carrasco, Pamies \& Narciso, 2012; Carrasco \& Gibson, 2010; Choi \& Calero, 2013; OCDE, 2013; Stanat \& Christensen, 2007); (2) la lengua materna de los escolares inmigrantes no establecerá diferencias en su comprensión lectora (García et al., 2008, 2015; Huguet et al., 2011; Huguet et al., 2007; Sansó et al., 2015); (3) existirán diferencias en esta competencia en función de la titularidad del centro (Carvallo et al., 2007; García et al., 2008, 2015; Gregorio \& Prado, 2016; Luna, 2011); (4) los años de residencia influirán positivamente en la comprensión lectora de los alumnos procedentes de otros países (Berry, 1990; Huguet \& Navarro, 2003; Navarro \& Huguet, 2006; Sansó et al., 2015). 


\section{METODOLOGÍA}

Se trata de un estudio descriptivo correlacional de tipo ex post facto, en el que hemos empleado análisis descriptivos e inferenciales de tipo multivariado y bivariado. Hemos comenzado realizando análisis multivariados (análisis multivariante de la varianza) para determinar la posible existencia de diferencias entre grupos en las variables de interés tomadas conjuntamente, en función de factores como la lengua de origen de los alumnos, la titularidad del centro y el tiempo de residencia en España. De este modo, si el estadístico de contraste (Lambda de Wilks) era significativo, procedíamos a realizar los contrastes bivariados (prueba $\mathrm{T}$ de Student para muestras independientes) para identificar en qué aspectos de la comprensión lectora existían diferencias significativas, así como análisis post hoc (Scheffé) para determinar qué grupos diferían significativamente. Además, hemos utilizado la correlación de Pearson para determinar la posible existencia de asociaciones significativas entre variables numéricas. El nivel de significación establecido en los diferentes análisis ha sido de alfa=.05.

\subsection{PARTICIPANTES}

La población objeto de estudio la constituyen los alumnos extranjeros que estudian ESO en Salamanca y el alumnado autóctono actúa como grupo de control. De este modo, los participantes consistieron en una muestra de conveniencia que aceptó voluntariamente participar en el estudio, tras garantizarse el anonimato y la confidencialidad de la información. Así, la muestra estuvo formada por 402 estudiantes, de los que un 52.2\% estaba constituido por mujeres. Del total, un $48.8 \%$ eran inmigrantes procedentes de 27 países, con mayor presencia de escolares latinoamericanos $(76.5 \%)$, con el castellano como lengua materna mayoritaria (72.4\%). En cuanto al tiempo de estancia en España, la mayoría llevaba de dos a siete años $(64.8 \%)$; fueron muy pocos los recién llegados o aquellos que llevaban residiendo en el país más de diez años. Por último, añadimos que los participantes estaban escolarizados en siete instituciones educativas, de las que cinco eran públicas, donde se ubicaba el $68.9 \%$ del alumnado inmigrante participante en la investigación.

\subsection{INSTRUMENTO}

Para la recogida de datos se realizó un análisis en profundidad de los instrumentos existentes que evaluaban la comprensión lectora, como el TPC (Martínez et al., 2008) o el SEDEC (Bel, Serra \& Vila, 1994). Sin embargo, estos cuestionarios se centran más en aspectos formales de la adquisición del idioma, obviando el componente sociocultural del mismo. Además, en su mayoría no están diseñados para educación secundaria y utilizan fundamentalmente preguntas cerradas. Otros, se basan en el diagnóstico de dificultades en la adquisición del lenguaje o en el aprendizaje de la lectura como competencia. Por tanto, optamos por diseñar un cuestionario ad hoc. Así, el instrumento está compuesto por seis preguntas abiertas referidas a un texto adjunto, diferente para cada curso, extraído de sus libros de Lengua Castellana y Literatura, con objeto de que los textos utilizados para la recogida de datos se relacionaran con el nivel educativo de los alumnos y, por tanto, con lo establecido en el currículo (Orrantia \& Sánchez, 1994). Para la elaboración 
de los ítems nos apoyamos en aquellos aspectos que estudiosos en el tema consideran los elementos fundamentales de una buena comprensión lectora (Alonso, 2005; Orrantia \& Sánchez, 1994; Pérez Zorrilla, 2005): el acceso al significado del vocabulario incluido en el texto (aspecto que viene determinado, entre otros factores, por el ámbito social y de procedencia de la persona, o su nivel cultural); las frases hechas en función del contexto; la temática del texto; la identificación de las partes en que se divide el mismo y su relación entre sí para, a partir de ahí, extraer la idea principal y realizar un resumen, relacionándolo con su experiencia propia y sus esquemas de conocimiento. En cada caso, el alumno tenía, en primer lugar, que indicar las palabras que le resultaban desconocidas, de las que posteriormente se realizaba un recuento que nos informaba sobre la cantidad de vocabulario que desconocían y que, ya de entrada, entorpecía su acceso a la totalidad de la información que el texto exponía. Las respuestas al resto de preguntas fueron baremadas en una escala de 1 a 4, (1-mal; 2-regular; 3-bien; 4-muy bien), y puntuadas por consenso entre un grupo de jueces expertos a partir de criterios previamente establecidos.

El cuestionario se sometió a un análisis de fiabilidad utilizando el coeficiente Alfa de Cronbach, en el cual se obtuvo un resultado de .79, lo que se puede considerar satisfactorio. Además, previamente fue administrado a una muestra de 91 alumnos de ESO con los que el instrumento ofreció pruebas de su fiabilidad y su validez discriminativa (Martín-Pastor, González-Gil \& Sánchez).

\subsection{PROCEDIMIENTO}

Para conocer los datos de población inmigrante que cursaba ESO en Salamanca y solicitar autorización para la realización del estudio, nos contactamos con la Dirección Provincial de Educación, que nos proporcionó las cifras de alumnos extranjeros en esta etapa y nos remitió al equipo directivo de cada centro.

La información recopilada nos permitió seleccionar aquellos centros que contaban en sus aulas con un mayor número de alumnos inmigrantes. Se estableció contacto con los mismos, en primer lugar, por correo electrónico, en el cual se les informaba del objetivo de la investigación y, posteriormente, vía telefónica para concertar entrevistas con el director, orientador y jefe de estudios de cada uno de ellos. Conseguidas las autorizaciones, se procedió conjuntamente a organizar y poner en marcha la evaluación en cada centro.

\section{RESULTADOS}

Para analizar si existían diferencias en comprensión lectora entre el alumnado inmigrante y el autóctono, tal y como plantea la primera hipótesis del estudio, se realizó, en primer lugar, un recuento del número de palabras desconocidas por ambos grupos. El análisis reveló diferencias significativas $(\mathrm{t}=-3,376, \mathrm{p}<0,05)$, de modo que los estudiantes extranjeros son los que presentan mayores problemas con el vocabulario $(\mathrm{M}=0,70)$, frente a sus iguales nacionales $(\mathrm{M}=1,33)$.

El análisis de las diferencia de medias en el resto de los ítems permitió constatar que también existían diferencias significativas en todas las preguntas. En la Tabla 1 se observa que la media de los alumnos españoles es significativamente superior en todos los casos a la obtenida por sus compañeros inmigrantes. 
Tabla 1. Comparación de medias en comprensión lectora (muestra total)

\begin{tabular}{|c|c|c|c|c|c|c|}
\hline Categoría & Ítem & Procedencia & $\mathrm{N}$ & Media & Des. típica & $\mathrm{t}$ \\
\hline \multirow{3}{*}{ Temática texto } & Ítem 2 & & & & & $12.936^{*}$ \\
\hline & & Español & 206 & 3.18 & .797 & \\
\hline & & Inmigrante & 196 & 2.01 & 1.008 & \\
\hline \multirow{3}{*}{ Estructura texto } & Ítem 3 & & & & & $14.013 *$ \\
\hline & & Español & 206 & 3.20 & .920 & \\
\hline & & Inmigrante & 196 & 1.84 & 1.028 & \\
\hline \multirow{3}{*}{ Idea principal } & Ítem 4 & & & & & $13.193 *$ \\
\hline & & Español & 206 & 2.89 & .979 & \\
\hline & & Inmigrante & 196 & 1.65 & .902 & \\
\hline \multirow{12}{*}{$\begin{array}{l}\text { Frases hechas, } \\
\text { expresiones } \\
\text { coloquiales }\end{array}$} & Ítem 5a & & & & & $10.565 *$ \\
\hline & & Español & 206 & 3.39 & .960 & \\
\hline & & Inmigrante & 196 & 2.20 & 1.273 & \\
\hline & Ítem $5 b$ & & & & & $13.639 *$ \\
\hline & & Español & 206 & 3.37 & .952 & \\
\hline & & Inmigrante & 196 & 1.95 & 1.131 & \\
\hline & Ítem 5c & & & & & $10.788^{*}$ \\
\hline & & Español & 206 & 3.12 & 1.146 & \\
\hline & & Inmigrante & 196 & 1.85 & 1.204 & \\
\hline & Ítem $5 \mathrm{~d}$ & & & & & $16.008 *$ \\
\hline & & Español & 206 & 3.50 & .865 & \\
\hline & & Inmigrante & 196 & 1.92 & 1.106 & \\
\hline \multirow{3}{*}{ Resumen } & Ítem 6 & & & & & $17.796 *$ \\
\hline & & Español & 206 & 2.92 & .735 & \\
\hline & & Inmigrante & 196 & 1.59 & .770 & \\
\hline
\end{tabular}

*Diferencias significativas con $\mathrm{p}<.01$

Fuente: elaboración propia.

Conforme a nuestra segunda hipótesis, se evaluaron las diferencias en comprensión lectora en función de la lengua materna de los alumnos inmigrantes para comprobar si constituía una variable que influía en sus dificultades. Para ello, los participantes fueron divididos en dos grupos: uno con hispanoparlantes y otro con estudiantes cuya lengua era diferente al castellano. Los análisis multivariados pusieron de manifiesto la existencia de diferencias significativas (Lambda de Wilks= .903; $\mathrm{F}_{(9.184)}=2.191 ; \mathrm{p}<.01$ ), por lo que se realizaron contrastes bivariados donde, como se muestra en la Tabla 2, solamente existen diferencias estadísticamente significativas $(\mathrm{p}<.05)$ entre ambos subgrupos en los ítems 1,2 y 5c. Esto nos permite adelantar que tener el castellano como primer idioma no es un factor que beneficie a los alumnos extranjeros en esta competencia, ni que se traduzca en menores dificultades en la misma. 
Estudios Pedagógicos XLIII, N²: 179-193, 2017

DIVERGENCIAS SOCIOCULTURALES EN EL USO DEL CASTELLANO: REPERCUSIONES EN LA COMPRENSIÓN DEL ALUMNADO INMIGRANTE EN EL SISTEMA EDUCATIVO ESPAÑOL

Tabla 2. Diferencias en comprensión lectora según lengua materna (muestra inmigrantes)

\begin{tabular}{|c|c|c|c|c|c|c|}
\hline Categoría & Ítem & Lengua materna & $\mathrm{N}$ & Media & Des. típica & $\mathrm{t}$ \\
\hline \multirow{3}{*}{ Vocabulario } & Ítem 1 & & & & & $-2.860 * *$ \\
\hline & & Castellano & 142 & 1.01 & 1.384 & \\
\hline & & No castellano & 54 & 2.17 & 4.251 & \\
\hline \multirow{3}{*}{ Temática texto } & Ítem 2 & & & & & $3.173 * *$ \\
\hline & & Castellano & 142 & 2.15 & 1.031 & \\
\hline & & No castellano & 54 & 1.65 & .850 & \\
\hline \multirow{3}{*}{ Estructura texto } & Ítem 3 & & & & & 1.005 \\
\hline & & Castellano & 142 & 1.89 & 1.053 & \\
\hline & & No castellano & 54 & 1.72 & .960 & \\
\hline \multirow{3}{*}{ Idea principal } & Ítem 4 & & & & & .706 \\
\hline & & Castellano & 142 & 1.68 & .911 & \\
\hline & & No castellano & 54 & 1.57 & .882 & \\
\hline \multirow{12}{*}{$\begin{array}{l}\text { Frases hechas, } \\
\text { expresiones } \\
\text { coloquiales }\end{array}$} & Ítem $5 \mathrm{a}$ & & & & & 1.008 \\
\hline & & Castellano & 142 & 2.26 & 1.259 & \\
\hline & & No castellano & 54 & 2.06 & 1.309 & \\
\hline & Ítem $5 b$ & & & & & .176 \\
\hline & & Castellano & 142 & 1.96 & 1.123 & \\
\hline & & No castellano & 54 & 1.93 & 1.163 & \\
\hline & Ítem $5 \mathrm{c}$ & & & & & $2.145^{*}$ \\
\hline & & Castellano & 142 & 1.96 & 1.234 & \\
\hline & & No castellano & 54 & 1.56 & 1.076 & \\
\hline & Ítem $5 d$ & & & & & 1.389 \\
\hline & & Castellano & 142 & 1.99 & 1.111 & \\
\hline & & No castellano & 54 & 1.74 & 1.085 & \\
\hline \multirow{3}{*}{ Resumen } & Ítem 6 & & & & & 1.602 \\
\hline & & Castellano & 142 & 1.64 & .793 & \\
\hline & & No castellano & 54 & 1.44 & .691 & \\
\hline
\end{tabular}

$* *$ Diferencias significativas con $\mathrm{p}<.01 ; *$ Diferencias significativas con $\mathrm{p}<.05$ Fuente: elaboración propia.

También se analizó la repercusión de la titularidad de los centros educativos en la comprensión lectora de los alumnos, de acuerdo con la tercera hipótesis formulada. Para ello, se realizó un análisis múltiple de varianza donde se interrelacionaron las variables: 
tipo de centro (público vs. concertado) y procedencia de los participantes (inmigrante vs. español). Los resultados desvelaron ausencia de diferencias en función de la titularidad $\left(\right.$ Lambda de Wilks $\left.=.958 ; \mathrm{F}_{(9.388)}=1.891 ; \mathrm{p}>.05\right)$. Sin embargo, el origen del alumnado afectaba significativamente las puntuaciones (Lambda de Wilks $=.425 ; \mathrm{F}_{(9.388)}=58.318$; $\mathrm{p}<.01$ ).

Por último, se estudió la influencia del tiempo de residencia en España en la comprensión lectora del alumnado inmigrante. Para ello, inicialmente se llevó a cabo un análisis correlacional en el cual se encontraron asociaciones significativas con dos categorías: vocabulario $\left(\mathrm{r}_{\mathrm{xy}}=-.189 ; \mathrm{p}<.01\right)$ e identificación de la estructura del texto $\left(\mathrm{r}_{\mathrm{xy}}=.143 ; \mathrm{p}<.05\right)$. De manera complementaria se realizaron contrastes bivariados que reflejaron la tendencia positiva de mejores resultados a partir del cuarto año de estancia en el país, aunque las diferencias solo resultasen estadísticamente significativas en los dos ítems señalados. Finalmente, los análisis post hoc (Scheffé) revelaron que el grupo de alumnos que llevaba de 1 a 3 años residiendo en España se diferenciaba sustancialmente del resto.

\section{DISCUSIÓN Y CONCLUSIONES}

Con el presente estudio hemos contribuido a aportar evidencias de las dificultades a las que se ha de enfrentar el alumnado extranjero. Así, los resultados indican que estos estudiantes obtienen puntuaciones más bajas en todos los ítems de comprensión lectora que el resto de sus compañeros. Dichas diferencias se concretan en primer lugar en un mayor desconocimiento del vocabulario incluido en el texto, aspecto fundamental que desde el principio puede condicionar la interpretación del contenido, ya que como señala Alanís (2004), las palabras son las herramientas que utilizamos para acceder al conocimiento, expresar ideas y aprender nuevos conceptos, lo que hace que el manejo del vocabulario por los alumnos esté estrechamente ligado a su éxito escolar. También Salvador et al. (2007) coinciden en que el dominio léxico favorece la comprensión lectora. De igual manera, Celedón-Pattichis (2004) desveló los problemas de los estudiantes hispanos bilingües en Estados Unidos para comprender el vocabulario en los enunciados de los problemas de matemáticas.

Junto a estas diferencias, puestas de manifiesto en estudios previos, encontramos además dificultades por parte del alumnado inmigrante para inferir el significado de las expresiones coloquiales. En este escenario, los escolares extranjeros cuentan con una desventaja respecto a sus compañeros, producto de la interacción con un texto escrito en otra cultura diferente a la suya y, por tanto, con unas prácticas discursivas distintas (Cassany, 2006). Lo anterior interfiere en la comprensión de estos alumnos, que obtienen además unas puntuaciones medias inferiores en los ítems restantes relativos a identificar la temática e idea principal del texto, su estructura, y elaborar un resumen de su contenido. En esta línea, estudios a nivel nacional e internacional (Choi \& Calero, 2013; Gregorio \& Prado, 2016; OCDE, 2013; Stanat \& Christensen, 2006) han señalado las diferencias que se dan en el rendimiento académico en lengua y matemáticas entre los escolares españoles e inmigrantes, de los cuales los segundos obtienen peores resultados en comprensión lectora. Datos que corroboran Carrasco y Gibson (2010) en un estudio comparativo sobre la situación del alumnado extranjero en España y EE. UU., y en ambos países, se apreciaron disparidades en los resultados académicos con respecto a sus compañeros autóctonos, siendo especialmente negativos los obtenidos por aquellos de origen latino y marroquí. 
Estudios Pedagógicos XLIII, Nº 2: 179-193, 2017

DIVERGENCIAS SOCIOCULTURALES EN EL USO DEL CASTELLANO: REPERCUSIONES EN LA COMPRENSIÓN DEL ALUMNADO INMIGRANTE EN EL SISTEMA EDUCATIVO ESPAÑOL

En cuanto a la influencia de la lengua de origen en el nivel de comprensión, cabe señalar que, aunque lo esperable es que poseer el castellano como lengua materna sea un elemento facilitador, los resultados, excepto en tres ítems, no muestran diferencias significativas entre los participantes hispanohablantes y aquellos cuyo primer idioma no es el castellano. Esto nos permite confirmar que las dificultades en comprensión lectora de los alumnos extranjeros no se justifican en su totalidad por el hecho de que hablen otra lengua (al describir a los participantes inmigrantes de esta investigación se indicó que un 72,4\% eran originarios de países de habla hispana), sino, más bien, por la cultura sobre la que se sustenta lo escrito y lo dicho. De hecho, Nusche (2009) afirma que solo hay medio curso educativo de diferencia entre los escolares inmigrantes cuya lengua materna es la misma que la lengua de instrucción de la escuela y los que no. Otras investigaciones relacionadas con este tema (Huguet et al., 2011; Huguet et al., 2007) han demostrado que los alumnos extranjeros, con independencia de que su idioma de origen sea o no el castellano, no llegan a igualar al autóctono en el dominio de la lengua a pesar de que su permanencia en España sea superior a seis años, lo que hace que esta variable no resulte determinante (Sansó et al., 2015). Profesores de EE. UU. destacan también que la mayoría de estos educandos que residen en el país, a pesar de llevar tres años o más, carecen del nivel de inglés que la educación requiere y asocia al trabajo académico (Carrasco \& Gibson, 2010).

Ante esta situación, nos cuestionamos las acciones que las administraciones educativas están llevando a cabo a través de los programas de inmersión lingüística, compensatorios y de enseñanza del español como segunda lengua (L2). Estas estrategias no hacen más que reducir la interculturalidad a cuestiones que únicamente conciernen a inmigrantes que desconocen por completo la lengua vehicular de la escuela, sin pararse a pensar qué ocurre con aquellos que son hispanoparlantes o que tienen un buen dominio del castellano (Besalú, 2011). De hecho, el objetivo prioritario para las aulas de acogida o aulas de compensatoria ha venido siendo la adquisición del idioma en el menor tiempo posible (Simó et al., 2014). Además, se trata de medidas que centran el aprendizaje de la competencia comunicativa en la adquisición de aspectos gramaticales y dejan al margen, en muchas ocasiones, el componente social y cultural que influye en el uso que se haga de una lengua en función del contexto donde esta se desarrolle (Prado, 2011). Por otro lado, se trata de acciones, metodologías y recursos que parecen responder más a la necesidad de defender a la escuela de la invasión del alumnado de otros contextos geográficos, culturales y lingüísticos, y la desestabilización que ello pueda provocar en el buen funcionamiento del sistema, que a la de conseguir que estos estudiantes disfruten de una educación de calidad en los mismos términos y con iguales oportunidades que sus compañeros (Besalú, 2011; Gregorio \& Prado, 2016). De hecho, en la evaluación que Arroyo y Rodríguez (2014) realizaron sobre las estrategias y aprendizajes conseguidos en la enseñanza de la lengua a escolares inmigrantes, resaltaron la postura de profesores que señalaban la falta de una planificación más seria y reflexiva sobre la finalidad de las medidas vigentes. Es decir, en palabras de Hernández y otros (2016) "las aulas interculturales son una realidad, otra cosa es que sean realmente interculturales e inclusivas" (p. 128).

En relación a los niveles de comprensión de los alumnos en función de la titularidad del centro en el que estaban matriculados, los resultados desvelan que esta variable no establece diferencias significativas, a pesar de la tendencia hacia puntuaciones más bajas en los centros públicos; este aspecto coincide con investigaciones previas (Carvallo et al., 2007) en las que se señala que los centros privados obtienen mejores puntuaciones 
en lengua y ciencias que los públicos, diferencias que podrían explicarse si comparamos los datos relativos a la presencia de alumnos inmigrantes en cada tipo de centro. Luna (2011), Carrasco et al. (2012) y García et al. (2008) advierten que los centros públicos escolarizan a un mayor número de inmigrantes que la enseñanza privada, puntualizando además que cuánto más elevado sea el porcentaje de estos alumnos en las aulas, peores son los resultados que se obtienen en las diferentes áreas curriculares. De hecho, como se constató en los resultados obtenidos, es la variable procedencia la que establece diferencias significativas en la comprensión lectora.

Si analizamos la información proporcionada por la Dirección Provincial de Educación de Salamanca, a la que están adscritos 10 centros públicos y 21 concertados de Educación Secundaria Obligatoria en la capital, se puede corroborar esta situación al observar no solo cómo el número de estudiantes extranjeros que cursan ESO es superior en la enseñanza pública $(60 \%)$, sino que además, el $40 \%$ restante se distribuye en un mayor número de centros, concentrado un $12 \%$ en dos de ellos, ambos participantes en esta investigación. A ello hay que añadir que, como señala Prado (2011), una de las soluciones por las que ha optado el sistema educativo haya sido la segregación en aulas de educación compensatoria de minorías étnicas o grupos raciales y culturales procedentes de otras zonas geográficas; se trata de una práctica alejada sustancialmente de los planteamientos de la inclusión que conciben la diversidad como una oportunidad para el enriquecimiento mutuo. Carrasco et al. (2012) advierten, ante esta situación, que una educación inclusiva no puede permanecer inalterable con aquellas actuaciones que puedan desembocar en menores oportunidades escolares y sociales para los educandos.

Otras investigaciones han estudiado la influencia de esta variable en los hábitos lectores de los alumnos (Gómez-Villalba \& Pérez, 2001) y afirman que los estudiantes de los centros concertados tienen una mayor actividad lectora, o la relación existente entre dichos hábitos y la comprensión lectora (Carvallo et al., 2007). De este modo si son los alumnos de los centros concertados los que invierten un mayor tiempo en la lectura, es predecible que ello se traduzca en una mejor comprensión lectora, según se refleja en los resultados presentados.

Por último, al considerar la influencia de la variable "tiempo de residencia en España", se puede observar que los participantes con más años de estancia en el país han obtenido mejores puntuaciones en todos los ítems que aquellos que llevaban menos de 3; sin embargo, estas diferencias únicamente resultan significativas en el dominio del vocabulario y en la identificación de la estructura del texto. Otros estudios relacionados con este tema (Huguet \& Navarro, 2003; Navarro \& Huguet, 2006; Navarro et al., 2016; Sansó et al., 2015) señalan que los estudiantes inmigrantes muestran un conocimiento adecuado de la lengua castellana y, por tanto, menos problemas, cuando su tiempo de estancia en el país supera los 6 años. En la misma línea, Cummins (2000 Cit. en Huguet, 2008) afirma que son necesarios un mínimo de 5 años para poder, en cierto modo, igualar a los nativos en los aspectos académicos de la lengua que son los que garantizan, en parte, el éxito escolar de estos alumnos. Por otro lado, consideramos como factor que puede intervenir en esta situación el fenómeno de la aculturización, entendida, en palabras de Berry (1990) como "el proceso mediante el cual las personas cambian, siendo influidas por el contacto con otra cultura" (p. 460). Algunos de los alumnos inmigrantes que participaron en este estudio manifestaron que paulatinamente habían tenido que cambiar su manera de comunicarse (aprender nuevas palabras y sus connotaciones, formas de expresarse, el acento) para poder 
Estudios Pedagógicos XLIII, Nº 2: 179-193, 2017

DIVERGENCIAS SOCIOCULTURALES EN EL USO DEL CASTELLANO: REPERCUSIONES EN LA COMPRENSIÓN DEL ALUMNADO INMIGRANTE EN EL SISTEMA EDUCATIVO ESPAÑOL

adaptarse mejor a la sociedad española, y comprender lo que les decían sus compañeros o lo que les enseñaban los profesores. De igual forma, los inmigrantes hispanos que participaron en el estudio de Schwieter (2011) en EE. UU. reconocieron haber sufrido presiones tanto sociales como culturales en la escuela y en la comunidad que afectaban negativamente a su compromiso escolar y que ellos tenían que "aculturizarse" para minimizar las diferencias con la cultura dominante.

Para finalizar, creemos necesario señalar algunas limitaciones y propuestas de mejora del presente estudio. En relación con el procedimiento de selección de los participantes, al dirigirnos únicamente a los centros con mayor concentración de inmigrantes, la muestra podría presentar un cierto sesgo, justificable en cierto modo, por el objetivo con el que enfocamos nuestro trabajo; así, al pretender analizar la situación del alumnado extranjero en el contexto educativo, consideramos fundamental centrarnos en aquellas instituciones donde más se concentraban, accediendo, de este modo casi a un $50 \%$ de la población inmigrante que cursaba ESO en Salamanca capital. No obstante, estudios posteriores nos permitirán ampliar la muestra, así como confirmar o refutar los presentes hallazgos. Igualmente interesante sería completar la investigación incluyendo aspectos contextuales, relacionados con variables como el nivel socioeconómico de los padres, la estructura familiar, la tipología de barrio, u otros factores que en el presente estudio no han podido ser incluidos. Pese a lo expuesto, consideramos que los resultados obtenidos suponen un avance en el análisis de las dificultades a las que se enfrenta el alumnado inmigrante y una oportunidad para reflexionar sobre la idoneidad de las repuestas que proporcionamos a sus necesidades. Todo ello con el objetivo de garantizar a cada alumno una educación que se adapte a sus características, a partir del trabajo colaborativo, la participación de la comunidad educativa y la implicación de la Administración.

\section{REFERENCIAS BIBLIOGRÁFICAS}

Alanís, I. (2004). Effective Instruction: Integrating Language and Literacy. In C. Salinas \& M. E. Franquiz (Eds.), Scholars in the Field: The Challenges of Migrant Education (pp. 211-224). Charleston: ERIC.

Alonso, J. (2005). Claves para la enseñanza de la comprensión lectora. Revista de Educación, número extraordinario, 63-93.

Alvarado, J. M., Puente, A., Jiménez, V., \& Arrebillaga, L. (2011). Evaluating Reading and Metacognitive Deficits in children and adolescents with Attention Deficit Hyeractivity disorder. Spanish Journal of psychology, 14(1), 62-73. doi:10.5209/rev_SJOP.2011.v14.n1.5

Arroyo, M. J., \& Rodríguez, M. (2014). Enseñanza de segundas lenguas a alumnado inmigrante: evaluación de las estrategias y aprendizajes conseguidos. Tendencias Pedagógicas, 24, 327-346.

Beacco, J.C., Byram, M., Cavalli, M., Coste, D., Cuenat, M.E., Goullier, F., \& Phantier, J. (2010). Guide for the development and implementation of curricula for plurilingual and intercultural education. Estrasburgo: Consejo de Europa.

Bel, A., Serra, J. M., \& Vila, I. (1994). Estudio comparativo del conocimiento de catalán en sexto, séptimo y octavo de EGB en 1990. En M. Siguan (Ed.), Las lenguas en la escuela (pp. 229-252). Barcelona: Horsori.

Berry, J. W. (1990). Psychology of Acculturation. In J. Berman (Ed.), Cross-cultural perspectives: Nebraska Symposium on Motivation (pp. 457-488). Lincoln: University of Nebraska.

Besalú, X. (2011). ¿Cómo defenderse del alumnado extranjero? En F. J. García Castaño y S. Carrasco 
(Eds.), Población inmigrante y escuela: conocimientos y saberes de investigación (pp. 81-92). Madrid: Ministerio de Educación, Secretaría General Técnica.

Bordieu, P., \& Thompson, J. B. (2011). Langage et pouvoir symbolique. Paris: Seuil.

Brind, T., Harper, C., \& Moore, K. (2008). Education for Migrant, Minority and Marginalised Children in Europe. Open Society Institute's Education Support Programme. Retrieved from https://www.opensocietyfoundations.org/sites/default/files/review_20080131.pdf

Carrasco, S., \& Gibson, M. A. (2010). La educación de jóvenes de origen inmigrante en secundaria: algunas lecciones comparativas desde Cataluña y California. Revista del Ministerio de Trabajo e Inmigración, 90, 59-76.

Carrasco, S., Pamies, J., \& Narciso, L. (2012). A propósito de la acogida de alumnado extranjero. Paradojas de la educación inclusiva en Cataluña (España). Revista Iberoamericana de Educación Inclusiva, 5(1), 105-122.

Carvallo, M., Caso, J., \& Contreras, L. A. (2007). Estimación del efecto de variables contextuales en el logro académico de estudiantes de Baja California. Revista Electrónica de Investigación Educativa, 9(2). Recuperado desde http://redie.uabc.mx/vol9no2/contenido-carvallo.html, $12 / 09 / 2012$

Cassany, D. (2006). Tras las líneas. Sobre la lectura contemporánea. Barcelona: Anagrama.

Celedón-Pattichis, S. (2004). Alternative Secondary Mathematics Programs for Migrant Students: Cultural and Linguistic Considerations. In C. Salinas \& M. E. Franquiz (Eds.), Scholars in the Field: The Challenges of Migrant Education (pp. 197-210). Charleston: ERIC.

Choi, A., \& Calero, J. (2013). Determinantes del riesgo de fracaso escolar en España en PISA2009 y propuestas de reforma. Revista de Educación, 362, 562-593. doi:10.4438/1988-592XRE-2013-362-242

Darmody, M., Byrne, D., \& McGinnity, F. (2014). Cumulative disadvantage? Educational careers of migrants students in Irish secondary schools. Race Ethnicity and Education, 17(1), 129-151.

García, F. J., Rubio, M., \& Bouachra, O. (2008). Población inmigrante y escuela en España: un balance de investigación. Revista de Educación, 345, 23-60.

García, F. J., Rubio, M., \& Bouachra, O. (2015) Immigrant students at school in Spain: constructing a subject of study. Dve domovini-Two Homelands, 41, 35-46.

Gómez-Villalba, E., \& Pérez, J. (2001). Animación a la lectura y comprensión lectora en Educación Primaria. Lenguaje y textos, 17, 9-20.

González, M. (1987). Lengua escolar y clase social. Salamanca: Amarú.

Gregorio, M., \& Prado, S. (2016). Dinámicas socioeducativas y construcción de imágenes del alumnado de origen inmigrante. Revista Internacional de Comunicación y Desarrollo, 3(1), 7190. doi:10.15304/ricd.1.3.2519

Hernández, M. A., Gomariz, M.A., Parra, J., \& García, M.P. (2016). Familia, inmigración y comunicación con el centros escolar. Un estudio comparativo. Educación XXI, 19(2), 127-151. doi:10.5944/educxx1.16457

Huguet, Á. (2008). Interdependencia lingüística y práctica educativa. Un estudio con escolares de origen inmigrante. Infancia y Aprendizaje, 31(3), 283-301. doi:10.1174/021037008785702901

Huguet, Á., \& Navarro, J. L. (2003). Inmigración y conocimiento de la lengua castellana: el caso de los escolares inmigrados en Aragón. Alazet: Revista de filología, 15, 343-364.

Huguet, Á., Navarro, J. L., \& Janés, J. (2007). La adquisición del castellano por los escolares inmigrantes. El papel del tiempo de estancia y la lengua familiar. Anuario de Psicología, 38(3), 357-375.

Huguet, Á., Chireac, S. M., Navarro, J. L., \& Sansó, C. (2011). Tiempo de estancia y aprendizajes lingüísticos. El caso de los escolares inmigrantes en Cataluña. Cultura y Educación, 23(3), 355370. doi:10.1174/113564011797330252

Kymlicka, W., \& Patten, A. (2003). Language Rights and Political Theory. Annual Review of Applied Linguistics, 23, 3-21. 
Estudios Pedagógicos XLIII, Nº 2: 179-193, 2017

DIVERGENCIAS SOCIOCULTURALES EN EL USO DEL CASTELLANO: REPERCUSIONES EN LA COMPRENSIÓN

DEL ALUMNADO INMIGRANTE EN EL SISTEMA EDUCATIVO ESPAÑOL

Leith, M. (2012). How Learning English Facilitates Integration for Adult Migrants: The Jarrah Language Centre Experience. Adelaide (Australia): National Centre for Vocational Education Research (NCVER).

Luna, F. (2011). Rendimiento educativo del alumnado inmigrante en las evaluaciones del sistema educativo vasco. En I. Ballano (Coord.), Evaluar la competencia lingüística del alumnado inmigrante (pp. 87-108). Bilbao: Deusto Digital, Universidad de Deusto.

Martín-Pastor, E., González-Gil, F., \& Sánchez Gómez, M. C. (2011). La inclusión del alumnado inmigrante y en situación de desventaja sociocultural. Evaluación de competencias lectoescritoras y comunicacionales. Revista de Educación Inclusiva, 4(2), 11-20.

Martínez, T., Vidal-Abarca, E., Sellés, P., \& Gilabert, R. (2008). Evaluación de las estrategias y los procesos de comprensión: el test de procesos de comprensión (TPC). Infancia y Aprendizaje, 31(3), 319-332.

Moreno, F. (2007). Adquisición de segundas lenguas y Sociolingüística. Revista de Educación, 343, $55-70$.

Navarro, J. L., \& Huguet, Á. (2006). Acerca de la competencia lingüística del alumnado de origen inmigrante en educación secundaria. Revista Interuniversitaria de formación de profesorado, $56,69-80$.

Navarro, J. L., Huguet, Á., \& Sansó, C. (2016). Procesos de interdependencia entre lenguas. El caso del alumnado inmigrante en Cataluña. Revista de Psicodidáctica, 21(2), 227-243. doi:10.1387/ RevPsicodidact.13396

Nusche, D. (2009). What Works in Migrant Education? A Review of Evidence and Policy Options. Paris: OCDE.

OCDE. (2013). PISA 2012. Programa para la evaluación internacional de los alumnos. Informe español. Volumen I: Resultados y Contexto. Madrid: Ministerio de Educación, Cultura y Deporte.

Oller, J., \& Vila, I. (2011). Variables asociadas al conocimiento del catalán y castellano escrito del alumnado extranjero. Un estudio empírico en sexto de primaria en Cataluña. Infancia y Aprendizaje, 34(4), 427-447.

Olson, D. R. (1998). El mundo sobre el papel. El impacto de la escritura en la estructura del conocimiento. Barcelona: Gedisa.

Orrantia, J., \& Sánchez, E. (1994). Evaluación del lenguaje escrito. En M. A. Verdugo (Dir.), Evaluación curricular: Una guía para la intervención psicopedagógica (pp. 223-326). Madrid: Siglo XXI.

Pérez, G., \& Sarrate, M. L. (2013). Diversidad cultural y ciudadanía. Hacia una educación superior inclusiva. Educación XXI, 16(1), 85-104. doi:10.5944/educXXI.16.1.718

Pérez Zorrilla, M J. (2005). Evaluación de la comprensión lectora: dificultades y limitaciones. Revista de Educación, número extraordinario, 121-138.

Prado, J. (2011). Didáctica de la lengua y la literatura para educar en el siglo XXI. Madrid: La Muralla.

Ruiz, L. (2000). Las metáforas de un día en los medios de comunicación españoles. Estudios de lingüística, 14, 199-216.

Sacristán, F. (2006). Comprensión lectora en alumnos de enseñanza secundaria. Revista de Enseñanza Universitaria, 27, 57-67.

Salvador, F., \& Gutiérrez, R. (2005). Atención educativa al alumnado con dificultades en lectura y escritura. Málaga: Aljibe.

Salvador, F., Gallego, J. L., \& Mieres, C. G. (2007). Habilidades lingüísticas y comprensión lectora. Una investigación empírica. Revista de Pedagogía, 59(1), 153-166.

Sánchez, A., Navas, L., \& Holgado, P. (2013). Inmigración y educación intercultural en la formación inicial docente. Estudios Pedagógicos, 39(1), 239-251.

Sansó, C., Navarro, J. L., \& Huguet, Á. (2015). La evolución del conocimiento lingüístico del alumnado inmigrante en Cataluña: el papel de la lengua familiar. Electronic Journal of Research 
in Educational Psychology, 13(2), 409-430. doi:10.14204/ejrep.36.14081

Santana, L.E., Feliciano, L., \& Jiménez, A. B. (2016). Apoyo familiar percibido y proyecto de vida del alumnado inmigrante de Educación Secundaria. Revista de Educación, 372, 35-62. doi:10.4438/1988-592X-RE-2015-372-314

Santos, C. M., \& Campo, M. E. (2008). Características diferenciales de la lectura en vista de los alumnos con baja visión en la Educación Secundaria Obligatoria. Integración: Revista sobre ceguera y deficiencia visual, 53, 7-24.

Schwieter, J. W. (2011). Migrant Hispanic Students Speak Up: Linguistic and Cultural Perspectives on Low Academic Attainment. Diaspora, Indigenous, and Minority Education, 5(1), 33-47.

Simó, N., Pamies, J., Collet-Sabé, J., \& Tort, A. (2014). La acogida educativa en los centros escolares en Cataluña: más allá de los recursos específicos para el alumnado de nueva incorporación. Revista Complutense de Educación, 25(1), 177-194.

Stanat, P., \& Christensen, G. (2006). Where immigrant students succeed. A comparative review of performance and engagement in PISA 2003. Paris: OCDE.

Van Dijk, T. (2003). Racismo y discurso de élites. Barcelona: Gedisa. 
